\title{
Research of temperature distribution in rooms with radiant heating systems
}

\author{
Ivan Voloshko ${ }^{1, *}$, and Vasiliy Ushakov ${ }^{1}$ \\ ${ }^{1}$ National Research Tomsk Polytechnic University, 634050 Tomsk, Russia
}

\begin{abstract}
The results of temperature measurements in model rooms of a closed type with a system of radiant heating are presented. The temperatures of different designs' surfaces in large-sized rooms, heated by a gas infrared emitter of light type, are established. Conclusions are drawn about the mechanism of the medium's heating, depending on the conditions of heat exchange, and also on providing worker's comfortable working conditions, when the medium is heated by a gas infrared emitter.
\end{abstract}

\section{Introduction}

The actual task of the present time is the effective using fuel and energy resources, which is confirmed by the adoption of appropriate regulatory and legal acts at the regional and federal levels.

Modern architectural and constructional solutions of industrial buildings' elements made of lightweight structures require the using of new energy-efficient and energy-saving heating systems. If in the development of project documentation for the construction of new production rooms, energy-efficient heating and ventilation systems are performed with using of modern building structures, then there are many problems with the compensation of heat losses by external walls when using traditional heating systems in large-sized production buildings built many years ago. They are characterized, as a rule, by huge, often empty production areas with thermo-technical properties that do not meet modern requirements. Heat supply of such facilities with the use of traditional heating appliances is inexpedient and impossible since this will lead to irrational energy costs and the inability to ensure a proper microclimate of workers.

Modern practice of heat supply shows that traditional convective heating systems can not effectively heat premises for periodic and short-term use; premises with a partially used area; significant height; distant from heat networks, as well as open and semi-open areas due to their inherent number of fundamental shortcomings.

The solution of tasks to ensure the thermal regime of industrial facilities and create an optimal microclimate in the work area can be the use of autonomous radiant heating systems, for example, gas infrared emitters (GIE) [1-3]. The application of GIE is inextricably linked with the need to study the thermal regimes of working zones in areas with multi-layered enclosing structures, roof and overall equipment in conditions of both open and closed rooms

\footnotetext{
* Corresponding author: iv_voloshko@mail.ru
} 
[1-6]. The purpose of present research is to register the temperature distributions of the surfaces of the rooms' enclosing structures, to subsequent develop the mathematical model, and, as a consequence, to predict the optimal conditions for the spatial location and angles slope of the GIE.

\section{Methods and research's results}

To achieve the delivered purpose, experimental plant (Fig. 1) was used, consisting of the main constructive elements: 1) gas infrared emitter - GIE-5 of light type manufactured by "Schwank" s company with a rated heat power of $5 \mathrm{~kW}$; 2) source of gas; 3) model of the heat supply's object; 4) thermocouples chromel-alumel type (junction's thickness is $0.08 \mathrm{~mm}$ and measurement's absolute error is $5 \%$, total relative systematic error is 4.6\%); 5) ADC ethernet chassis cDAQ 9181 manufactured by "National Instruments" s company to control the timing, synchronization and transfer of data from a 16-channel, 32-bit isothermal temperature measurement module NI 9214 manufactured by "National Instruments" company; 6) PC.

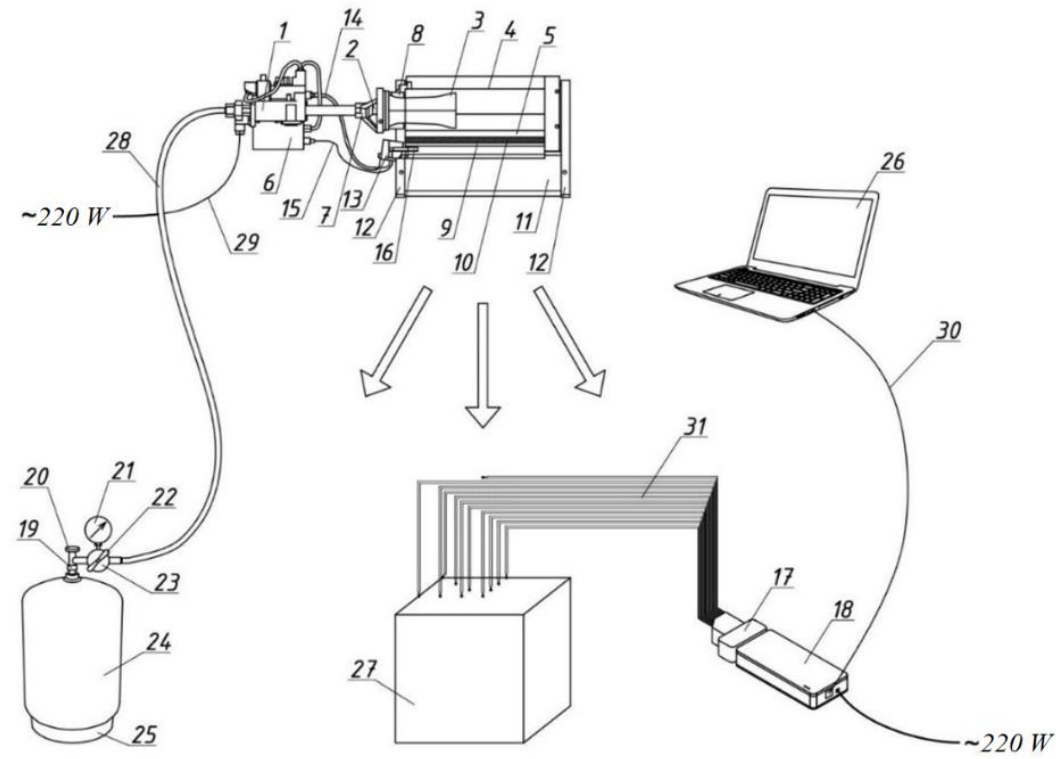

Fig. 1. The scheme of the experimental plant: 1 - valves block, 2 - nozzle, 3 - mixing tube, 4 - gas burner body, 5 - spreaders, 6 - infrared control, 7 - coupling, 8 - connecting tube, 9 - grids, 10 - ceramic tiles, 11 - reflectors, 12 - face fetlock, 13 - protective casing, 14 - high-voltage wire, 15 - flame sensor wire, 16 - spark plug with flame sensor, 17 - temperature measurement module NI-9214, 18 - ethernet chassis CompactDAQ Ethernet cDAQ-9181, 19 - valve, 20 - flywheel, 21 - output pressure manometer, 22 - adjusting screw, 23 - universal regulable reduction gear, 24 - propane gas cylinder's body, 25 - support, 26 - PC, 27 - model of the heat supply's object, 28 - connecting hose, 29 - electrical cable, 30 - connecting cable (twisted pair), 31 - thermocouples.

The experimental plant was located in two enclosed premises with different floor surfaces (white ceramic tiles and black concrete), with overall dimensions $10.2 \times 4.9 \times 4.4$ and $8.7 \times$ $6.0 \times 3.4 \mathrm{~m}$, height from floor to the lower edges of the reflector GIE -2.975 and $2.6 \mathrm{~m}$ respectively. The walls were manufactured from brick with a thickness of $70 \mathrm{~cm}$ with plastic window openings. The initial air temperature in the room ranged from 7 to $18{ }^{\circ} \mathrm{C}$, while the ambient temperature varied from -15 to $-35^{\circ} \mathrm{C}$. During the experiments the GIE was in a stationary position at controlled distances from the walls and in height. The thermocouples 
were placed on the floor surface and oriented along two coordinate directions (Fig. 2). The beginning of coordinate axes was centre of GIE. The distances (R) between two adjacent thermocouples of the same direction were $0.2 \mathrm{~m}$.

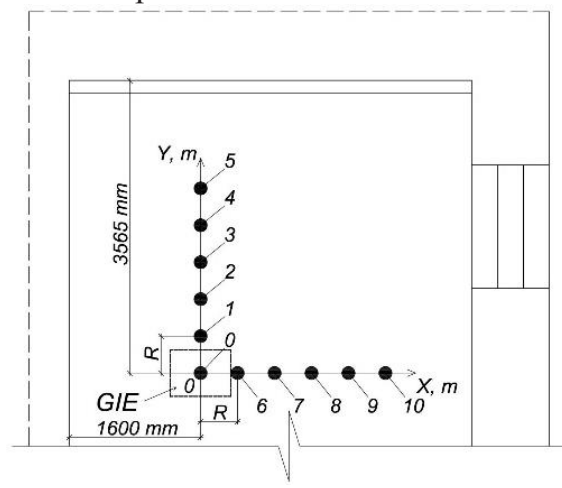

a)

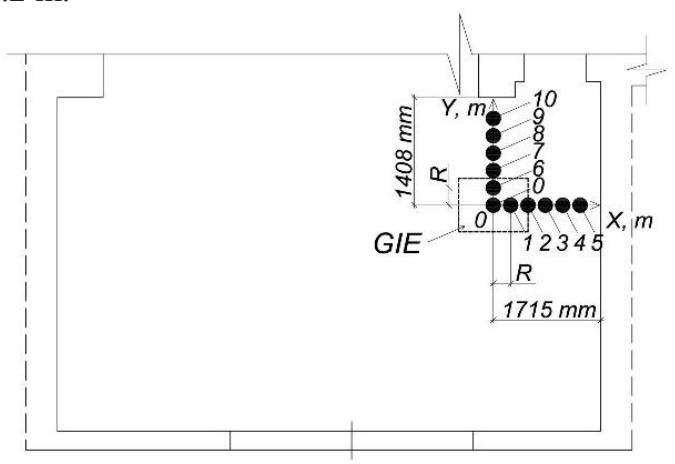

b)

Fig. 2. The schemes of placing GIE and thermocouples in experimental premises with different floor surfaces and geometric dimensions (the numerals correspond to the numbers of the thermocouples): a) - with ceramic tiles and dimensions $10.2 \times 4.9 \times 4.4 \mathrm{~m}$; b) - with a concrete floor and dimensions $8.7 \times 6.0 \times 3.4 \mathrm{~m}$.

The typical temperature distributions on different surfaces of the floor in time are shown in Fig. $3,4$.

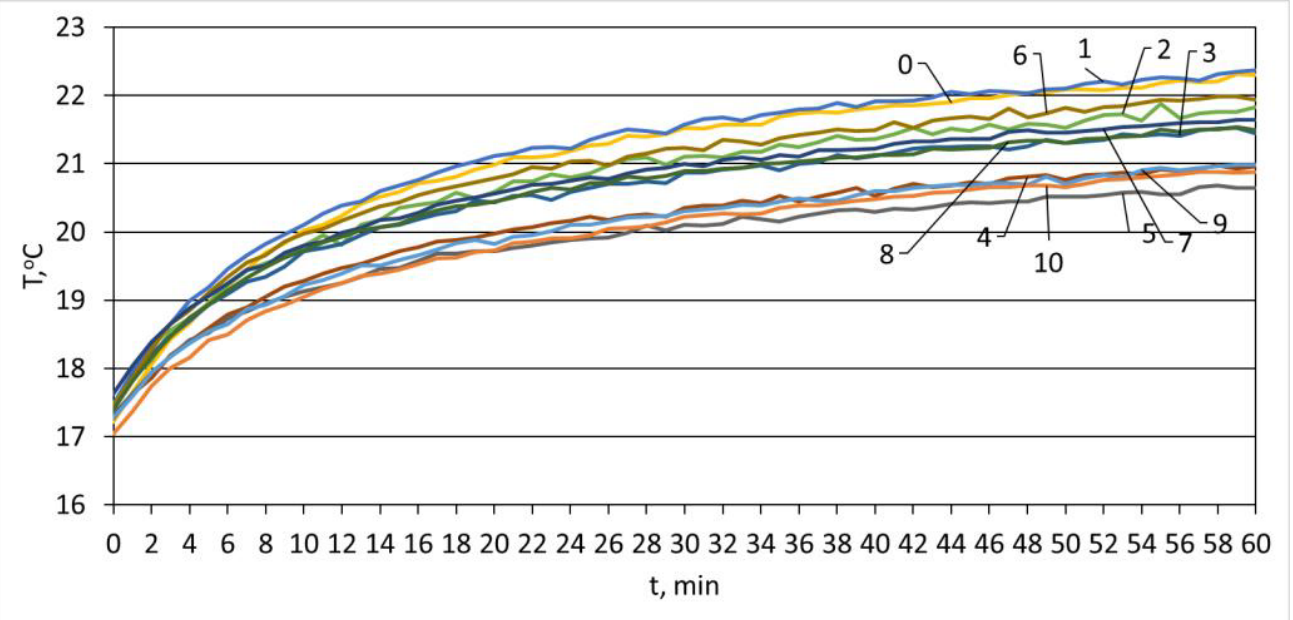

Fig. 3. Change of temperature in time at different points of the floor surface with ceramic tiles (the numerals correspond to the numbers of the thermocouples).

As can be seen from Fig. 3-4, the dynamics of changing the fields' temperatures of floor in time for different premises with different floor surface is the same. The maximum temperature of the floor surface is reached under the centre of the GIE. Then, as the thermocouples are moved away from the centre, the temperature values decrease unevenly. This unevenness is due to the peculiarity of the ceramic matrix's radiation used in the GIE. 50 minutes after the GIE switch-on, the temperature in the central region of the floor in both cases becomes $22{ }^{\circ} \mathrm{C}$. 


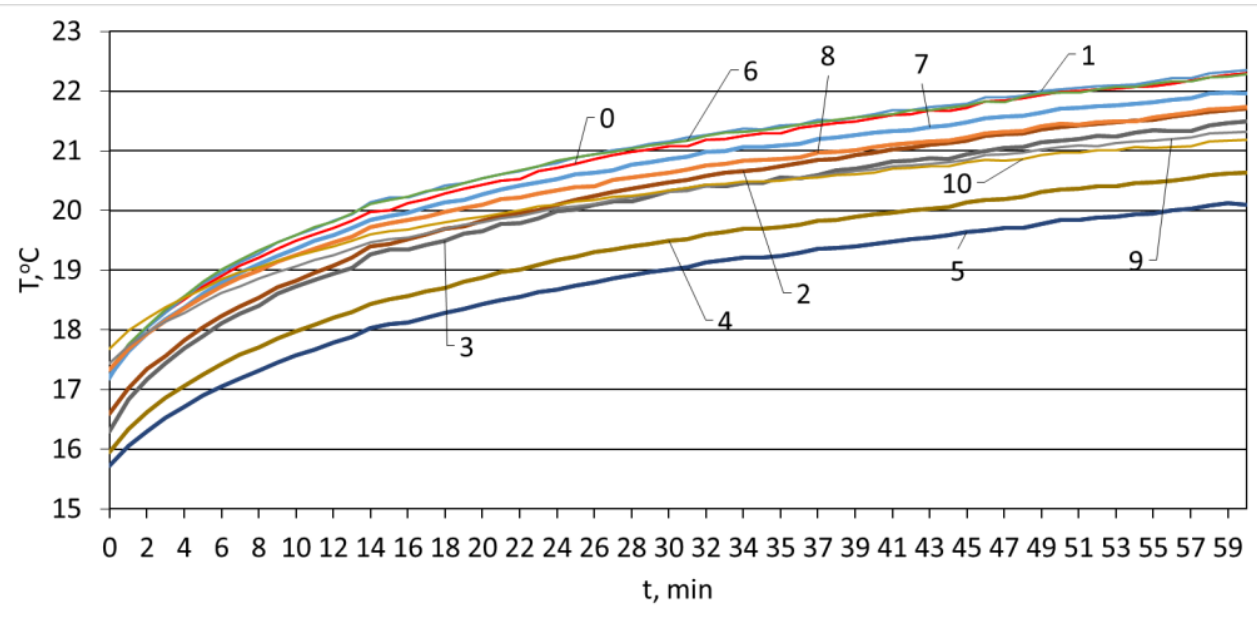

Fig. 4. Change of temperature in time at different points of the concrete floor surface (the numerals correspond to the numbers of the thermocouples).

This allows us to conclude that the temperature distribution on the floor surface of a room heated by a gas infrared emitter does not depend on the characteristic of the radiation of the floor's surface in the optical range. In the infrared range, the emissivity's coefficient of ceramic tiles and concrete is the same and equal to 0.95 . The regularities established in the experiments make it possible to formulate the hypothesis that the energy transfer in the "emitter-air-floor surface" system is most likely due to all three mechanisms of heat transfer (radiation, conduction and convection). It can also be concluded that the change in the geometric characteristics of large-sized rooms (volume) by $20 \%$ practically does not affect the temperatures' distribution on the floor surface heated by GIE.

The obtained experimental data can be used to improve the methods for calculating thermal regimes of used and designed large-sized premises heated by gas infrared emitters, as well as for developing and verification mathematical models of calculating the thermal regimes of large-sized premises heated by infrared emitters.

\section{References}

1. G. V. Kuznetsov, N. I. Kurilenko, V. I. Maksimov, G. Ya. Mamontov, T. A. Nagornova, J. Eng. Phys. Thermophys. 1 (2013)

2. G. V. Kuznetsov, N. I. Kurilenko, G. Ya. Mamontov, L. Yu. Mikhailova, EPJ Web. Conf. 82, (2015)

3. V. I. Maksimov, T. A. Nagornova, EPJ Web Conf. 76, (2014)

4. A. N. Ermolaev, S. A. Khaustov, MATEC Web Conf. 110, (2017)

5. M. Montiel-Gonzales, Appl. Therm. Eng. 75, 1176 (2015)

6. Q.-q. Li, C. Chen, Y. Zhang, J. Lin, H.-s. Ling, Y. Ma, Build Simul. 7, 207 (2014) 